\title{
Letter to the Editor Evidence for PDGFRA, PDGFRB and KIT deregulation in an NSCLC patient
}

\author{
T Negri', P Casieri', F Miselli', M Orsenigo', C Piacenza', S Stacchiotti' ${ }^{2}$ P Bidoli', PG Casali², MA Pierotti', \\ E Tamborini' and S Pilotti*,I \\ 'Laboratory of Experimental Molecular Pathology, IRCCS Istituto Nazionale dei Tumori, Via Venezian, I 20133 Milan, Italy; ${ }^{2}$ Department of Medical \\ Oncology, IRCCS Istituto Nazionale dei Tumori, Via Venezian, I 20133 Milan, Italy; ${ }^{3}$ RCCS Istituto Nazionale dei Tumori, Via Venezian, I 20133 Milan, \\ Italy
}

British Journal of Cancer (2007) 96, 180-181. doi:I0.1038/sj.bjc.6603542 www.bjcancer.com

(c) 2007 Cancer Research UK

Sir,

Vlahovic et al, provided evidence of effectiveness of Imatinib treatment in PDGFRB-overexpressing human lung adenocarcinoma xenografts grown in nude mice. In this preclinical model, the effectiveness of the drug is related to decrease in interstitial fluid pressure (IFP), decrease of phosphorylated PDGFRB and VEGF expression and improvement of oxygenation (Vlahovic et al, 2006). Furthermore, according to the working hypothesis that inhibition of PDGFRB decreases IFP and improves first-line therapy through drug uptake increase (Pietras, 2004), a clinical trial investigating the role of Imatinib as an adjuvant to chemotherapy is ongoing in stromal PDGFRB expressing non-small-cell lung cancer (NSCLC) (Vlahovic et al, 2006). Here we demonstrate that Imatinib in adjunction to chemotherapy may also be effective in a lung adenocarcinoma made up of tumour cells expressing Imatinib-sensitive deregulated genes such as PDGFRA, PDGFRB and $c$-Kit in presence of EGFR and HER-2/neu amplification lacking any evidence of protein expression.

An NSCLC, corresponding to a poorly differentiated adenocarcinoma, was diagnosed 1 month after starting treatment with Imatinib for a locally advanced sacral chordoma in a 64-year-old male patient. The chordoma expressed an activated PDGFRB (detected by immunoprecipitation/Western blotting (IP/WB) experiments) and the cognate ligand (by RT-PCR), findings consistent with the presence of an autocrine/paracrine loop (Casali et al, 2004). At the time the second primary was detected, the chordoma was showing a weak dimensional response to Imatinib.

The lung carcinoma was initially surgically treated, but relapsed to the mediastinum 17 months later, when the chordoma seemed to undergo slight secondary progression on Imatinib. Soon after stopping Imatinib, the patient received cisplatin plus vinorelbine with concomitant radiotherapy and, four months later, at the end of chemo-radiotherapy, CT and PET revealed an optimal complete radiological and functional response of lung tumour. Surprisingly, given the poor chemosensitivity of chordomas, there was also an initial minor response of the sacral tumour, which was lost soon after, but then was restored with the resumption of Imatinib (the

*Correspondence: Dr S Pilotti; E-mail: silvana.pilotti@istitutotumori.mi.it plan was to reintroduce the latter in combination with cisplatin, but renal function did not allow it).

The primary lung tumour was investigated in addition to FISH analysis, by IHC, IP/WB and DNA sequencing (after the informed consent of the patient) (Figure 1). Cumulatively, the FISH findings were consistent with a high degree of genetic instability showing high polisomy of PDGFRA, PDGFRB and $c$-Kit genes, coupled with activation (phosphorylation) of all the three receptors at IP/WB analysis and positivity at IHC (anti-PDGFRA sc-338 and anti-PDGFRB sc-339, Santa Cruz Biotechnology, CA, USA; anti-CD117 A4502, Dako, Carpinteria, CA, USA), as well as, as already anticipated, EGFR and HER-2/neu amplification coupled with null IHC (Dako) and biochemical analysis. Molecular analysis performed for investigating the mutation hot-spots of RTK genes excluded the presence of any activating mutation.

Finally, FISH analysis performed on the chordoma biopsy obtained during surgery for adenocarcinoma showed a dysomic pattern of all the investigated RTK genes.

The present findings imply firstly that the lung adenocarcinoma tumoral cells, in addition to EGFR and HER-2/neu gene deregulation, successfully treated with EGFR family inhibitors (Bunn et al, 2006), may harbour gene amplification and phosphorylation of Imatinib-sensitive RTKs, and so the spectrum of deregulated RTK genes in NSCLC is wider than believed. Secondly that, although supported by different mechanisms (an autocrine/paracrine activation loop of PDGFRA, PDGFRB and KIT in chordoma (Tamborini et al, 2006) and a gene gain in lung cancer), the two tumours carried the same Imatinib-sensitive deregulated genes and are thus expected to be sensitive to the same drug. The sequence of clinical events is hard to interpret but, together with the biomolecular findings, what was observed strongly supports the possibility that the efficacy of the chemotherapy against the NSCLC may have benefited from Imatinib, and the efficacy of Imatinib against chordoma may have benefited from the chemotherapy. Cumulatively, all these findings give further support to the ongoing clinical trial in selected patients with NSCLC (Vlahovic et al, 2006) and, in particular, for PDGFRB, they point out that this receptor is a multifaced player that through different mechanisms may affect the treatment response of both standard treatments and target therapies. 
FISH analysis:

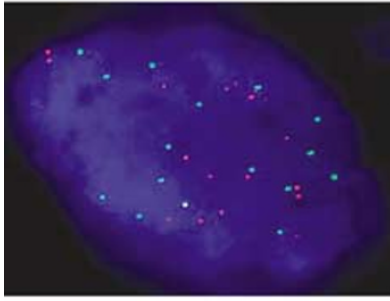

PDGFRB

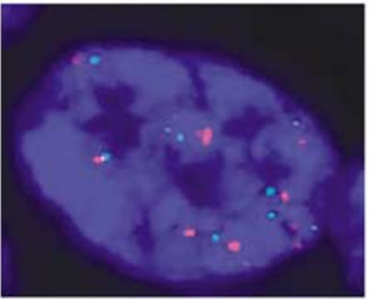

PDGFRA

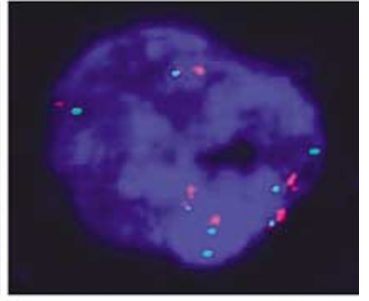

c-Kit

Immunoprecipitation experiments:

WB:

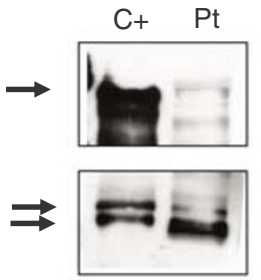

IP: Anti-PDGFRB

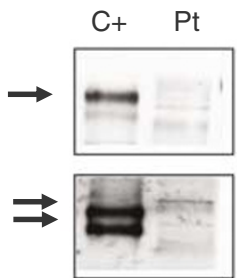

IP: Anti-PDGFRA

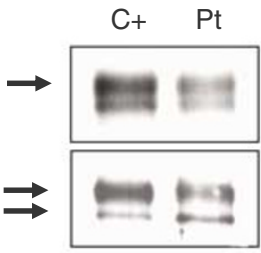

IP: Anti-KIT

Figure I Upper panel. FISH analysis. A probe mix for PDGFRB (BAC clone RPI I-368O I9) (in green)/a locus specific in 5p (in red), PDGFRA (BAC clone RPI I-23ICI8) (in green)/Cep4 (in red) (Vysis) and c-Kit (BAC clone RPI I-586A2 (in green)/Cep4 (in red) (Vysis), respectively, was employed to detect gene status for the receptors. A condition of high polisomy was detected for PDGFRB, PDGFRA and c-Kit. Lower panel. Immunoprecipitations/Western blot analysis. Equal amounts of total protein extracts (I mg) were immunoprecipitated using the specified antibodies (PDGFRB sc 432, Santa Cruz Biotechnology; PDGFRA 07-276, Upstate, Charlottesville, VA, USA and KIT Ab-3, clone K45, Neomarkers, Fremont, CA, USA) subsequently blotted to a membrane and incubated first with anti p-Tyr antibody (clone 4G, Upstate) and then with antibodies for the specified receptors. As positive controls, for PDGFRA and PDGFRB, an NIH3T3 cell line was employed, whereas for KIT a $\Delta 559$ cell line expressing a constitutively activated KIT receptor was used.

\section{ACKNOWLEDGEMENTS}

SP has research grants from Novartis; PGC received honoraria from Novartis. This paper was supported by grants from the AIRC
(Associazione Italiana per la Ricerca sul Cancro) and the Italian Ministry of Health (Ricerca Finalizzata 2004) (to Dr Pilotti).

\section{REFERENCES}

Bunn Jr PA, Dziadziuszko R, Varella-Garcia M, Franklin WA, Witta SE, Kelly K, Hirsch FR (2006) Biological markers for non-small cell lung cancer patient selection for epidermal growth factor receptor tyrosine kinase inhibitor therapy. Clin Cancer Res 12: $3652-3656$

Casali PG, Messina A, Stacchiotti S, Tamborini E, Crippa F, Gronchi A, Orlandi R, Ripamonti C, Spreafico C, Bertieri R, Bertulli R, Colecchia M, Fumagalli E, Greco A, Grosso F, Olmi P, Pierotti MA, Pilotti S (2004) Imatinib mesylate in chordoma. Cancer 101: 2086-2097

Pietras K (2004) Increasing tumor uptake of anticancer drugs with imatinib. Semin Oncol 31: 18-23
Tamborini E, Miselli F, Negri T, Lagonigro MS, Staurengo S, Dagrada GD, Stacchiotti S, Pastore E, Gronchi A, Perrone F, Carbone A, Pierotti MA, Casali PG, Pilotti S (2006) Molecular and biochemical analysis of PDGFRB, PDGFRA and KIT receptors in chordomas. Clin Cancer Res 12(23): $6920-6928$

Vlahovic G, Rabbani ZN, Herndon II JE, Dewhirst MW, Vujaskovic Z (2006) Treatment with Imatinib in NSCLC is associated with decrease of phosphorylated PDGFR-beta and VEGF expression, decrease in interstitial fluid pressure and improvement of oxygenation. Br J Cancer 95: $1013-1019$ 